\title{
Phosphatidylinositol-3 kinase signaling controls survival and stemness of hematopoietic stem and progenitor cells
}

\author{
Sasja Blokzijl-Franke ${ }^{1}$ Bas Ponsioen ${ }^{1,2}$. Stefan Schulte-Merker ${ }^{1,3} \cdot$ Philippe Herbomel $^{4,5} \cdot$ Karima Kissa $^{4,5,6}$. \\ Suma Choorapoikayil ${ }^{1,6} \cdot$ Jeroen den Hertog $\mathbb{D}^{1,7}$
}

Received: 10 September 2020 / Revised: 17 February 2021 / Accepted: 24 February 2021 / Published online: 13 March 2021

(c) The Author(s) 2021. This article is published with open access

\begin{abstract}
Hematopoietic stem and progenitor cells (HSPCs) are multipotent cells giving rise to all blood lineages during life. HSPCs emerge from the ventral wall of the dorsal aorta (VDA) during a specific timespan in embryonic development through endothelial hematopoietic transition (EHT). We investigated the ontogeny of HSPCs in mutant zebrafish embryos lacking functional pten, an important tumor suppressor with a central role in cell signaling. Through in vivo live imaging, we discovered that in pten mutant embryos a proportion of the HSPCs died upon emergence from the VDA, an effect rescued by inhibition of phosphatidylinositol-3 kinase (PI3K). Surprisingly, inhibition of PI3K in wild-type embryos also induced HSPC death. Surviving HSPCs colonized the caudal hematopoietic tissue (CHT) normally and committed to all blood lineages. Single-cell RNA sequencing indicated that inhibition of PI3K enhanced survival of multipotent progenitors, whereas the number of HSPCs with more stem-like properties was reduced. At the end of the definitive wave, loss of Pten caused a shift to more restricted progenitors at the expense of HSPCs. We conclude that PI3K signaling tightly controls HSPCs survival and both up- and downregulation of PI3K signaling reduces stemness of HSPCs.
\end{abstract}

\section{Introduction}

Stem cells define a particular type of cells that maintain selfrenewal capacity and may differentiate into multiple cell types at the same time. Hematopoietic stem and progenitor cells (HSPCs) are multipotent cells giving rise to all blood lineages during life [1-3]. In all vertebrates, an initial primitive wave of hematopoiesis occurs in the embryo, giving rise to primitive erythrocytes and myeloid cells. A definitive

Supplementary information The online version contains supplementary material available at https://doi.org/10.1038/s41388021-01733-5.

$\triangle$ Jeroen den Hertog

j.denhertog@hubrecht.eu

1 Hubrecht Institute-KNAW and University Medical Center Utrecht, Utrecht, The Netherlands

2 Molecular Cancer Research, Center for Molecular Medicine, University Medical Center Utrecht, Utrecht University, Utrecht, The Netherlands

3 Institute for Cardiovascular Organogenesis and Regeneration, Medical Faculty, WWU Münster, Münster, Germany wave follows in which HSPCs are generated that will found multi-lineage hematopoiesis in developmentally successive hematopoietic organs up to adulthood. Our understanding of the emergence of HSPCs during the definitive wave is derived primarily from pioneer live in vivo imaging [4-6]. HSPCs emerge in a process whereby cells in the ventral wall of the dorsal aorta (VDA) undergo an endothelial to hematopoietic transition (EHT) [5] and then transiently colonize the fetal liver in mammals [7], or the caudal hematopoietic tissue (CHT) in zebrafish [8]. There, HSPCs expand and differentiate into all blood lineages and supply the developing embryos with mature blood cells. Subsequently, HSPCs migrate again to colonize the thymus and the bone marrow in

4 Department of Developmental and Stem Cell Biology, Institut Pasteur, Paris, France

5 CNRS, UMR 3738, Paris, France

6 Laboratory of Pathogen Host Interaction, CNRS, INSERM, Univ Montpellier, Montpellier, France

7 Institute of Biology, Leiden University, Leiden, The Netherlands 
mammals [7] or whole kidney marrow in fish [8] to produce blood cells in the adult.

HSPCs are tightly regulated in terms of dormancy, selfrenewal, proliferation, and differentiation. Disrupting this balance can have pathological consequences such as bone marrow failure or hematologic malignancy. The tumor suppressor, PTEN, has an important role in hematologic malignancies, particularly $\mathrm{T}$-lineage acute lymphoblastic leukemia (T-ALL). Deleterious mutations in PTEN appear in $5-10 \%$ of T-ALL cases and about $17 \%$ of patients lack PTEN expression in the hematopoietic lineage $[9,10]$. PTEN counteracts phosphatidylinositol-3 kinase (PI3K) and hence acts upstream in the PI3K-Akt (also known as Protein kinase B) pathway [11]. Loss of PTEN function results in hyperactivation of the PI3K-Akt signaling pathway. Clonal evolution of leukemia-propagating cells in zebrafish highlights the role of Akt signaling in the process [12]. Conditional deletion of Pten in mice in hematopoietic stem cells (HSCs) in adult bone marrow promotes HSC proliferation, resulting in depletion of longterm HSCs, indicating that Pten is essential for the maintenance of HSCs [13, 14].

The zebrafish genome encodes two pten genes with redundant function designated ptena and ptenb [15]. Single mutants display no morphological phenotype and are viable and fertile, but mutants that retain only a single wild-type copy of pten develop hemangiosarcomas during adulthood [16]. Ptena ${ }^{-/}$ptenb $^{-/-}$mutants lack functional Ptena and Ptenb, are embryonic lethal at 5-6 days postfertilization (dpf), and display hyperplasia and dysplasia [15]. We reported that double mutant zebrafish larvae lacking functional Pten display increased numbers of HSPCs in the CHT at 4-5 dpf. Whereas these HSPCs commit to different blood lineages, they fail to differentiate into mature blood cells. Inhibition of PI3K using LY294002, which compensates for the loss of Pten, restores differentiation of HSPCs into mature blood cells. Hence, loss of Pten enhances HSPCs proliferation and arrests differentiation [17].

The past decades have led to an increase in our knowledge of hematopoiesis, but we are still far from a complete understanding of how HSPCs are established. Likewise, the role of Pten in steady-state hematopoiesis has been studied, but its potential role in the ontogeny of HSPCs is not fully understood. We have addressed these questions in zebrafish larvae. We imaged the emergence of HSPCs from the VDA in vivo in ptena ${ }^{-/-}$ptenb ${ }^{-/-}$ embryos and in PI3K inhibitor treated wild-type embryos, which showed surprisingly similar defects. Furthermore, we performed single-cell RNA sequencing (scRNA-seq) during the onset and at the end of the definitive wave. Our results indicate that elevated and reduced PI3K signaling had opposite effects on HSPCs at the end of the definitive wave.

\section{Results}

\section{The onset of the definitive wave of hematopoiesis is independent of Pten}

The onset of the definitive wave starts at $32 \mathrm{~h}$ postfertilization (hpf) with the specification of endothelial cells that will become HSPCs in the floor of the dorsal aorta (DA) in the AGM region (Fig. 1a), a conserved process between mammals and zebrafish [4-6]. Runxl expression from $32 \mathrm{hpf}$ onwards and $c-m y b$ expression from $35 \mathrm{hpf}$ onwards mark the hemogenic endothelium of the VDA and its HSPC progeny $[8,39]$. We found that ptena ${ }^{-/-}$pten ${ }^{-/-}$mutant embryos expressed runxl and $c-m y b$ along the VDA during the period that HSPCs emerge (between 30 and $44 \mathrm{hpf}$ ) just like their siblings (Fig. S1), indicating that loss of Pten does not affect the number of hemogenic endothelial cells.

\section{Loss of Pten results in apoptosis of HSPCs during EHT in ptena $^{-/}$ptenb $^{-/-}$mutant embryos}

In zebrafish, endothelial cells from the VDA transform into HSPCs in a process called EHT [5]. Subsequently, HSPCs join the blood flow in the underlying posterior cardinal vein to transiently seed the CHT $[4,5,8]$. We monitored EHT events in the AGM by time-lapse confocal imaging of an area spanning two adjacent intersegmental vessels in the $\operatorname{tg}(\mathrm{kdrl}$ : $e G F P$ ) transgenic background from 35 to $48 \mathrm{hpf}$ (Fig. 1a, b). The vasculature of pten ${ }^{-/-}$pten $^{-1-}$ mutants and siblings was indistinguishable at this stage [40]. The floor of the aorta in ptena $^{-/-}$pten $^{-/-}$mutant embryos displayed the characteristic contraction then bending of cells toward the subaortic space [5], indicating that the initiation of EHT was normal in ptena ${ }^{-/-}$ptenb $^{---}$mutant embryos. However, half of the EHT events in ptena ${ }^{-/-}$ptenb $^{-/-}$mutant embryos were abortive, in that 13 out of 24 HSPCs ( $54 \%$ in 9 embryos) failed to detach and disintegrated (Fig. 1c-h and Movie S1). In contrast, siblings or wild-type embryos did not display abortive EHT ( $n=75$ in total). Live imaging using acridine orange [30] revealed apoptotic cells in the DA of ptena ${ }^{-/-}$ptenb ${ }^{-/-}$mutant embryos, but not siblings (Fig. 1i-1). Activated caspase-3 immunostaining [31] confirmed apoptosis of $k d r l: e G F P$-positive cells at the VDA in ptena ${ }^{-/}$pten $^{-/-}$mutant embryos (Fig. $1 \mathrm{~m}-\mathrm{p}$ ). Hence, about half of the HSPCs in ptena ${ }^{-/-}$pten ${ }^{-/-}$mutant embryos failed to complete EHT and instead underwent apoptosis.

\section{The number of HSPCs that colonize the $\mathrm{CHT}$ is reduced in ptena ${ }^{-/-}$pten $^{-/-}$mutant embryos}

Following EHT, HSPCs transiently colonize the CHT [8]. We generated a $\operatorname{tg}(k d r l: D e n d r a 2)$ transgenic line. The Dendra2 protein along the entire VDA was 
wildtype
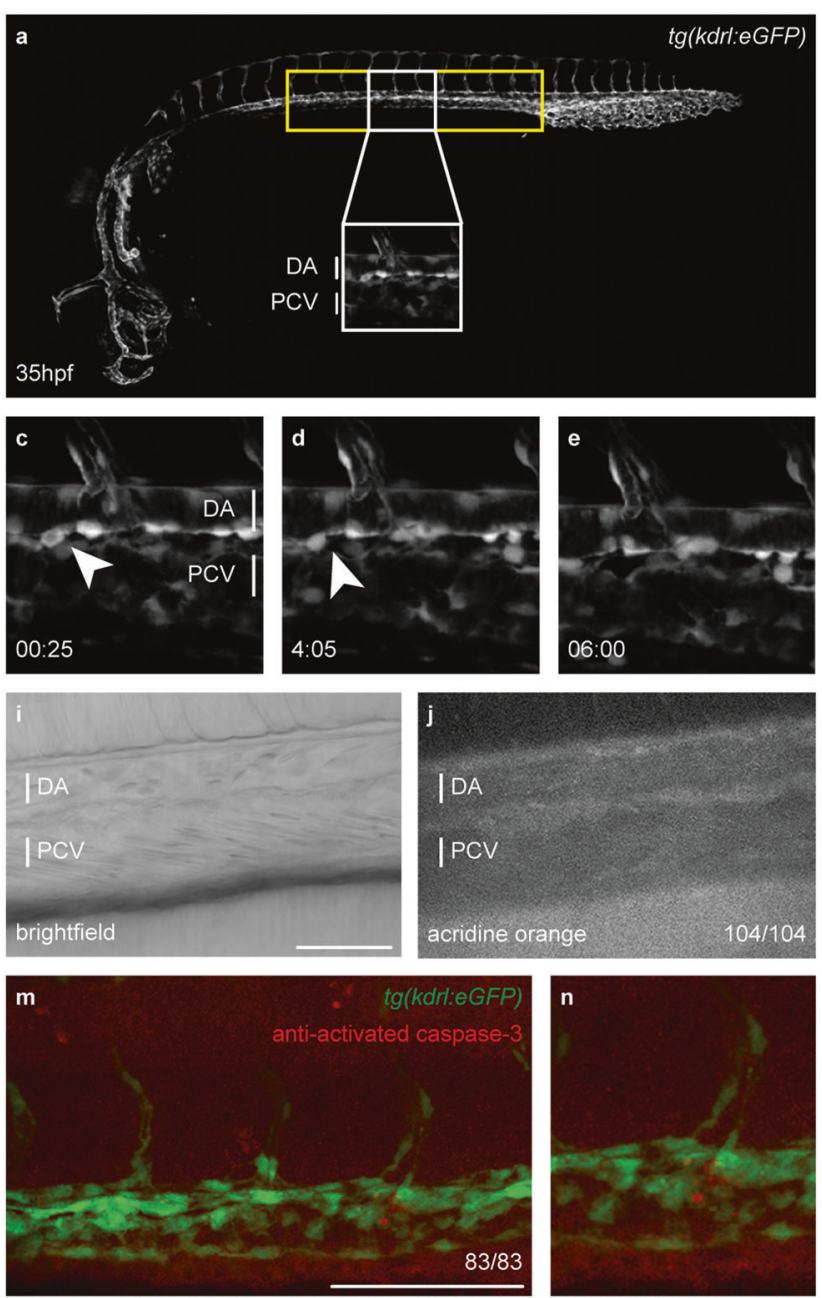

Fig. 1 A population of HSPCs fails to complete EHT and undergoes apoptosis in ptena $^{-/}$ptenb $^{-/-}$mutant embryos. a, b Brightfield image of a wild-type or pten ${ }^{-/-}$pten $^{-/-}$mutant zebrafish embryo at $35 \mathrm{hpf}$. The area from which HSPCs originate is indicated with a yellow box. A close up is indicated with a white box. c-h Fourdimensional imaging of $\operatorname{tg}(\mathrm{kdrl}: e G F P)$ wild-type or ptena $^{-/-}$ptenb $^{-/-}$ mutant embryos between 35 and 48 hpf. Still frames from Movie S1. Arrowheads: HSPCs undergoing EHT; asterisk: disintegrating HSPCs. Confocal image $\mathrm{z}$-stacks $(2 \mu \mathrm{m}$ step size, with $\times 40$ objective and $\times 2$ zoom; anterior to the left; maximum projections of a representative embryo; time in hh: mm. i-l Acridine orange staining. Arrows and circles: HSPCs in VDA of 40-45 hpf embryos. Asterisks: apoptotic

photoconverted green-to-red between 26 and $28 \mathrm{hpf}$, i.e. before the onset of EHT events (Fig. 2a). Photoconverted HSPCs in ptena ${ }^{-1}$ pten $^{-1-}$ mutant embryos colonized the CHT between 50 and $60 \mathrm{hpf}$, albeit less HSPCs were detected than in the CHT of siblings (Fig. 2b, c). We quantified the number of HSPCs that colonized the CHT at $48 \mathrm{hpf}$, i.e. by the peak of HSPC emergence from the VDA, using $\operatorname{tg}(c d 41: e G F P)$ embryos, which express low GFP $\left(\mathrm{GFP}^{\text {low }}\right)$ in HSPCs $[20,41]$. Consistent with the ptena ${ }^{-/ p t e n b^{-1}}$
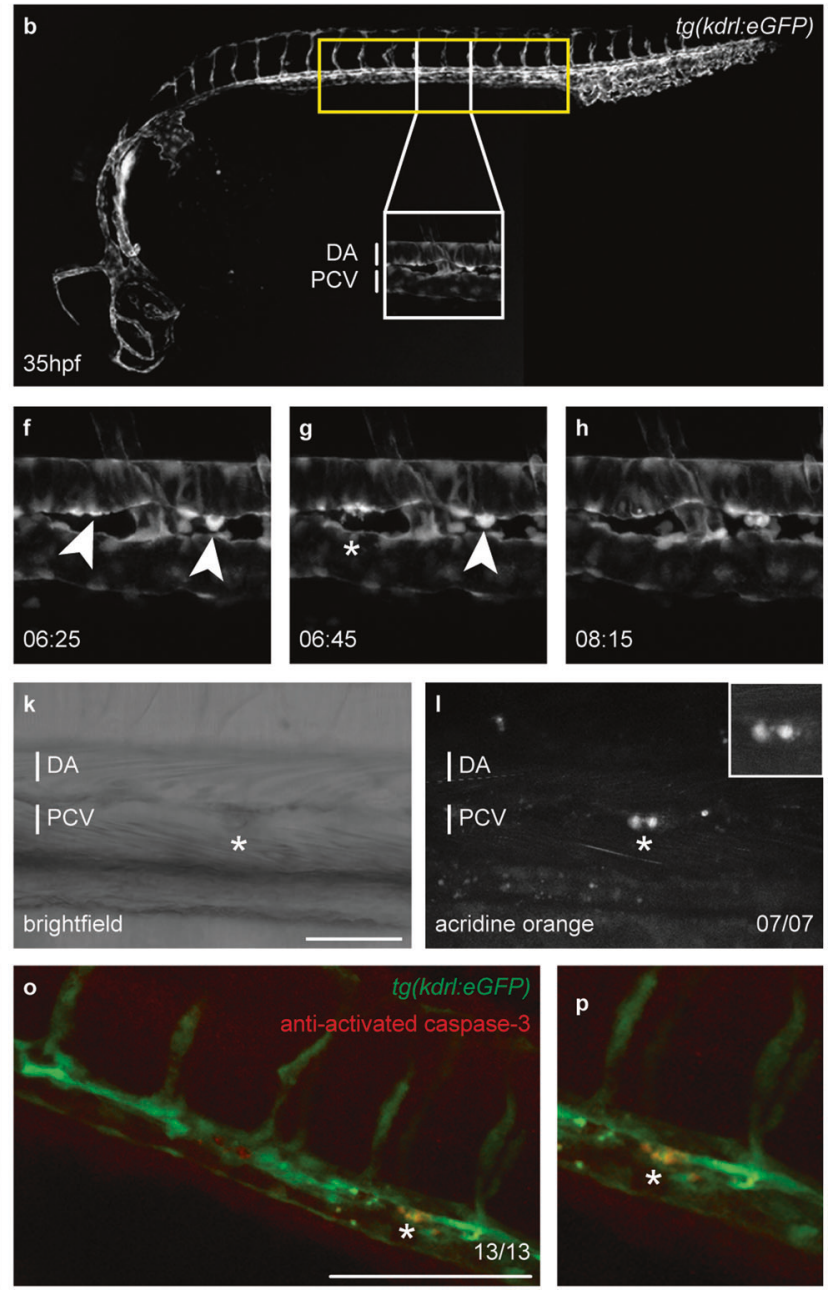

HSPCs. Scale bar: $50 \mu \mathrm{m}$. Representative embryos are shown and the number of embryos that showed this pattern/total number of embryos is indicated. DA dorsal aorta, PCV posterior cardinal vein. $\mathbf{m}-\mathbf{p}$ confocal images of apoptotic endothelial cells in the VDA of fixated wild-type or ptena ${ }^{-/-}$ptenb $^{-1-}$ mutant zebrafish embryos. In green: $\operatorname{tg}(k d r l: e G F P)$; in red: anti-activated caspase-3 immunohistochemistry staining. Apoptotic cells are indicated with an asterisk. Representative embryos are shown and the number of embryos displaying this particular pattern/total number of embryos is indicated in the bottom right. Anterior to the left; $2 \mu \mathrm{m}$ step size; maximum projections; scale bar: $100 \mu \mathrm{m}$.

initial apoptosis of half of the EHT-derived HSPCs, $51 \%$ less GFP $^{\text {low }}$ HSPCs were detected in the CHT of ptena ${ }^{-/}$pten $^{-/-}$mutant embryos at $48 \mathrm{hpf}$ compared to siblings (Figs. $2 \mathrm{~d}-\mathrm{f}$ and $\mathrm{S} 2$ ). When injected with ptenbmRNA at the one-cell stage, ptena ${ }^{-/-}$ptenb $^{-/-}$mutant embryos did no longer show a significant loss of HSPCs compared to their siblings (Fig. $2 \mathrm{~g}$ ), indicating that the observed defects indeed were caused by loss of functional Pten. 
Fig. 2 Less HSPCs colonize the CHT in ptena $^{-/-}$ptenb $^{-/-}$ mutant embryos than in wildtype. a The VDA of $t g(k d r l$ : Dendra2) was photoconverted green-to-red at 26-28 hpf. By 50-60 hpf red HSPCs derived from the photoconverted VDA had colonized the CHT in b sibling and $\mathbf{c}$ ptena ${ }^{-1}$ ptenb $^{-/-}$ larvae. d The number of GFP ${ }^{\text {low }}$ HSPCs at $48 \mathrm{hpf}$ in the CHT of $t g$ (cd41:eGFP) siblings (sib) $(n=25)(\mathbf{e})$ and ptena $^{-1-}$ ptenb $^{-/-}$ mutants (mut) $(n=12)(\mathbf{f})$ is expressed as average number of cells in siblings $(n=41)$ or ptena ${ }^{-/}$ptenb $^{-/}$. Mutants $(n=18)(\mathbf{g})$ is expressed as average number of cells in siblings $(n=33)$ or ptena $^{-/}$ptenb $^{-/-}$mutants after injection with synthetic ptenbmRNA $(n=15)$. Error bars indicate standard error or the mean (SEM). Shapiro-Wilk test for normal distribution and Welch's two-tailed $t$-test were used for statistical analysis; $* * * p<0.001$. Representative embryos are shown and the number of embryos that showed this pattern/total number of embryos is indicated.
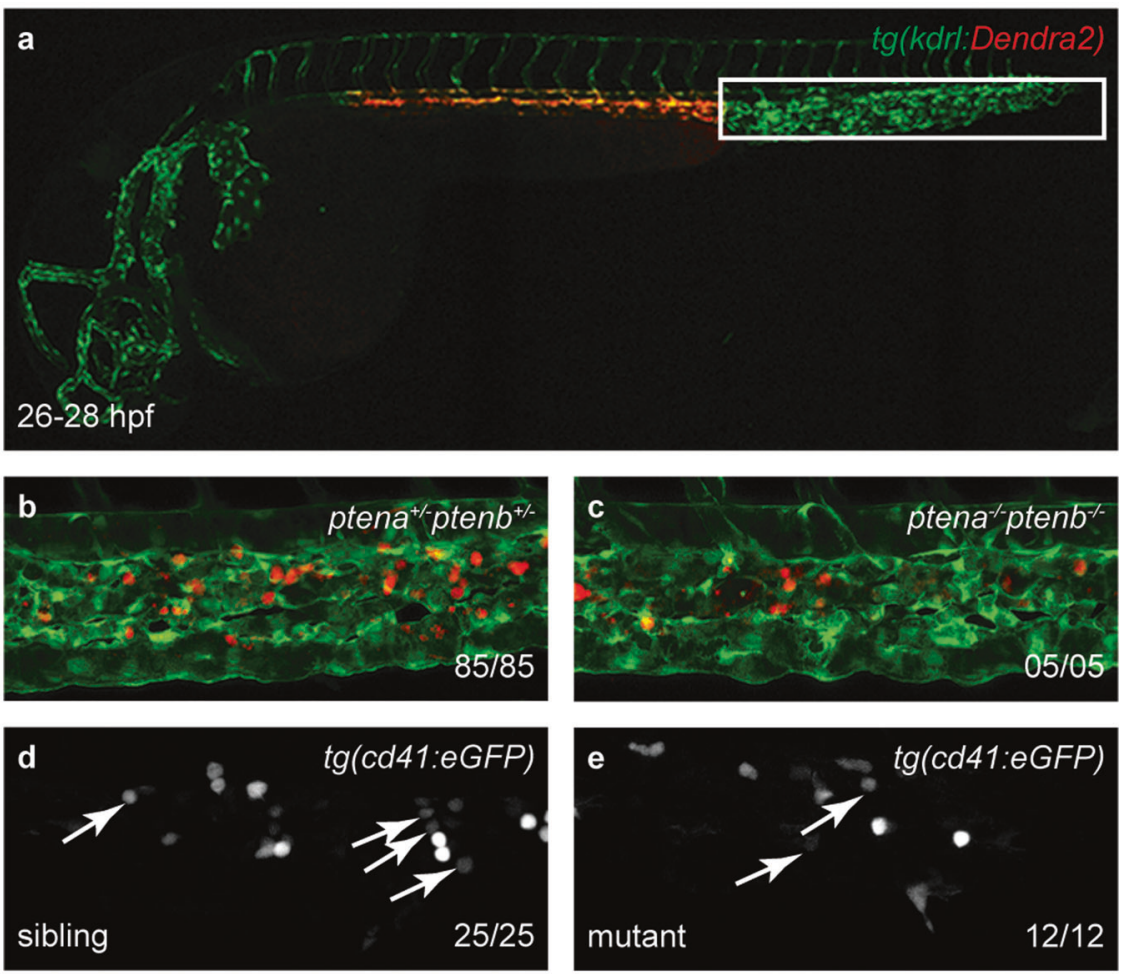

f

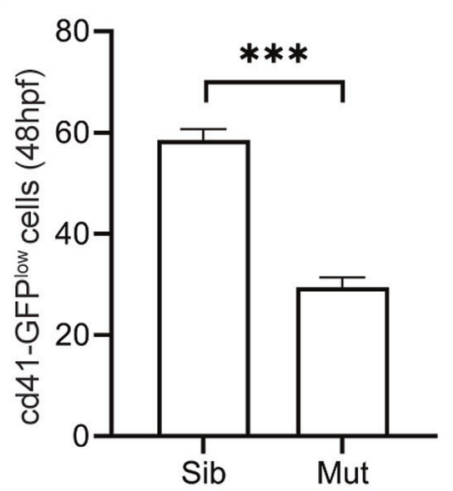

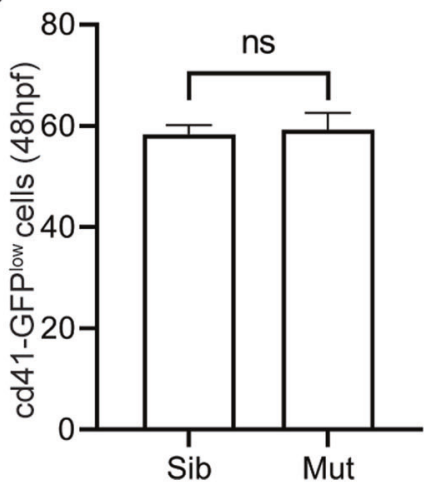

\section{PI3K inhibition rescues EHT events in ptena ${ }^{-/-}$ pten $^{-/-}$mutant embryos but is detrimental for HSPCs in wild-type embryos}

To address whether apoptosis of half of the EHT-derived HSPCs was due to enhanced PI3K signaling, embryos were treated with the PI3K inhibitor LY294002 from the onset of EHT (32 hpf) onwards. Inhibition of PI3K prevented apoptosis of HSPCs in ptena ${ }^{-1}$ ptenb $^{-/-}$mutant embryos, in that none of the HSPCs that we imaged disintegrated (Table 1) (Fisher's exact test, $p=0.0013$ ) (Fig. 3a-c). Surprisingly, in wild-type and sibling embryos that were treated with LY294002 in parallel with the ptena ${ }^{-/-}$ pten ${ }^{-1-}$ mutant embryos, disintegrating HSPCs in the VDA were observed $(n=6)$ (Fig. $3 \mathrm{~d}-\mathrm{g}$ and Movie S2) (Fisher's exact test, $p=0.021$ ).
Consistent with abortive EHT events upon LY294002 treatment, significantly less GFP ${ }^{\text {low }}$ HSPCs in $t g(c d 41: e G F P)$ transgenic embryos colonized the CHT of LY294002-treated wild-type embryos at $50 \mathrm{hpf}$ (Fig. 3h, i, 1), comparable to the reduction observed in ptena ${ }^{-/-}$ptenb ${ }^{-/}$mutant embryos (Fig. 2d). This reduction of HSPCs persisted through $4 \mathrm{dpf}$ in LY294002-treated embryos (Fig. 3j, k, m). These data suggest that normal, i.e. not too high and not too low PI3K activity levels are essential for emergence of HSPCs.

\section{PI3K inhibition in wild-type embryos results in HSPCs that engage in all blood lineages}

In situ hybridization was performed using a panel of blood progenitor markers. LY294002 treatment reduced expression of the HSPC marker $c-m y b$ at $4 \mathrm{dpf}$ (Fig. 4a, b). 
Table 1 Number of apoptotic cells during EHT.

\begin{tabular}{|c|c|c|c|c|c|}
\hline & \multirow[t]{2}{*}{ Embryos $(n)$} & \multicolumn{4}{|l|}{ HSPCs } \\
\hline & & Apoptosis & Escapers & \%Apoptosis & \% Escapers \\
\hline \multicolumn{6}{|l|}{ Wild-type } \\
\hline Control & 13 & 0 & 24 & 0 & 100 \\
\hline LY294002 & 6 & 6 & 9 & 40 & 60 \\
\hline \multicolumn{6}{|c|}{ ptena $^{-/-}$pten $^{-/-}$} \\
\hline Control & 9 & 13 & 11 & 54 & 46 \\
\hline LY 294002 & 5 & 0 & 21 & 0 & 100 \\
\hline
\end{tabular}

The number of cells undergoing apoptosis during EHT was determined in control and LY294002 (5 $\mu \mathrm{M}$ from $32 \mathrm{hpf}$ onwards) treated wild-type and ptena ${ }^{-1-}$ pten $^{-1-}$ embryos (35-48 hpf) by confocal time-lapse imaging. The number of embryos that was imaged is given as well as the number of apoptotic HSPCs or surviving HSPCs. The percentages of emerging or apoptotic HSPCs relative to the total number of HSPCs are also indicated.
The lineage markers globin (erythroid lineage), ikaros (lymphoid lineage), and l-plastin (pan-leukocytic, including myeloid lineage) were expressed, but reduced in LY294002-treated larvae compared to controls (Fig. 4c-h). LY294002-treated HSPCs also committed to the thrombocytic lineage as demonstrated by $\mathrm{GFP}^{\text {high }}$ cells in $\operatorname{tg}(c d 41$ : $e G F P$ ) embryos at $5 \mathrm{dpf}$ [20] (Fig. 4i, j). The number of $\mathrm{GFP}^{\text {low }}$ cells in the definitive hematopoietic organs, thymus, and kidney of 8 and $12 \mathrm{dpf} \operatorname{tg}(c d 41: e G F P)$ larvae was reduced in response to LY294002 (Fig. 4k-n). These results show that reduced PI3K signaling did not block specification of particular blood lineages, but that the reduction in HSPC numbers affected founding of the definitive hematopoietic organs by HSPCs.

\section{Singe-cell RNA sequencing reveals two types of HSPCs, one of which is preferentially lost upon inhibition of the PI3K-pathway}

To investigate transcriptomic changes in HSPCs between LY294002-treated embryos and their controls during EHT, we performed scRNA-seq. Transgenic $\operatorname{tg}(k d r l: m C h e r r y-$ $C A A X / c d 41: e G F P$ ) embryos were treated with LY294002 and AGM regions were isolated by dissection at $36 \mathrm{hpf}$. The AGM regions of 2000 control embryos were pooled and likewise, 2000 LY294002-treated embryos. The cells were dissociated and sorted for $\mathrm{mCherry}^{+} / \mathrm{eGFP}^{\text {low }}$ using FACS, after which the SORT-Seq protocol was performed [34] (Fig. S3). Isolation of 3219 cells in total, i.e. $<1 \mathrm{kdrl}^{+} / \mathrm{cd} 41^{\text {low }}$ $\left(\mathrm{mCherry}^{+} / \mathrm{GFP}^{\text {low }}\right.$ ) HSPC per embryo, was in line with earlier reports (3 HSPCs per embryo per hour [5, 42]). After FACS filtering, 2512 cells remained. RaceID3 [43] was used for differential gene expression analysis and clustering of the cells (Fig. 5). The resulting t-SNE map highlighted particular cell types, in line with recent scRNA-seq studies of hematopoietic organs of zebrafish [44-51], which expressed validated hematopoietic lineage markers (Table S1). Cells in clusters 2 and 4 expressed HSPC-related genes, such as gata2b, gfilaa, meis $1 b$, myb, and pmp22b, consistent with expression in mammalian HSCs and zebrafish HSPCs [45-49, 51] (Fig. 5a, b). RaceID3 subdivided the main HSPC cluster into two, HSPCs I and HSPCs II. Expression of ENSDARG00000080337_ACO24175.4 and tmed1b was higher in the HSPCs II cluster (cl2) than the HSPCs I cluster (cl4) (Figs. 5h and S3) and the expression of several other genes was also significantly different between these clusters (Fig. S4). Cells in cluster 5 expressed endothelial transcripts, that are known to be involved in the EHT-process ( $c d h 5$ [52, 53], adgrg1 [44, 54]), indicating an EHT progenitor lineage (Figs. 5c and S3). Signature genes $m p x$, lyz, marco, and mfap4 were expressed in clusters 1 and 3 [55], indicating a myeloid/neutrophil- and myeloid/monocyte-progenitor lineage, respectively (Figs. 5d, e and S3). All markers that were used to identify clusters are listed in Table S2 and the distribution of expression of selected markers is depicted in Fig. S5.

Cells from LY294002-treated embryos had an uneven distribution over all clusters. In HSPCs II and myeloid/ neutrophil progenitors, cells from LY294002-treated embryos were overrepresented compared to control embryos (Fisher's exact test, $p<0.001$ ). In HSPCs I, EHTand myeloid/monocyte progenitors, cells from LY294002treated embryos were underrepresented (Fisher's exact test, $p<0.001$ ) (Fig. 5f, i). These data indicate that LY294002 treatment led predominantly to loss of cells from HSPCs I cluster, which is consistent with the imaging data where half of the HSPCs fail to complete EHT (Fig. 3d-g). The loss of HSPCs I in response to PI3K inhibition is accompanied by an increase in HSPCs II and myeloid/neutrophil progenitors.

\section{More HSPCs upon inhibition of PI3K and less HSPCs in pten mutants}

The CHTs of 100 control and 100 LY294002-treated embryos were processed for scRNA-seq. Of the $928 \mathrm{~cd} 41^{\text {low }}$ 
Fig. 3 Inhibition of PI3K rescued EHT in ptena ${ }^{-/}$ ptenb $^{-/-}$mutant embryos, but induced abortive EHT in wildtype embryos. a-g Fourdimensional imaging of $t g(\mathrm{kdrl}$ : $e G F P$ ) transgenic embryos. a-c ptena ${ }^{-/}$pten $^{-/-}$mutant embryos and $\mathbf{d}-\mathbf{g}$ wild-type embryos. Imaging was done from $35 \mathrm{hpf}$ onwards following treatment with $5 \mu \mathrm{M}$ LY294002 from $32 \mathrm{hpf}$. Still frames from movie S3 (a-c) and movie S4 (d-g). arrowheads: HSPCs. Asterisks: disintegrating HSPCs. Different colors of arrowheads distinguish separate EHT events. Images were taken with $\times 40$ objective and $\times 1$ zoom. Time in hh:mm; DA dorsal aorta, PCV posterior cardinal vein. $\mathbf{h}, \mathbf{i}$ CHTs of $t g(k d r l: m C h e r r y-$ CAAX/cd41:eGFP) control $(n=$ 10) and LY294002-treated $(5 \mu \mathrm{M}, 32-50 \mathrm{hpf})(n=16)$ embryos were imaged at $50 \mathrm{hpf}$. The vasculature is highlighted in red (mCherry) and some GFPlow HSPCs are indicated by arrows. $\mathbf{j}, \mathbf{k}$ CHTs of $\operatorname{tg}(\mathrm{cd} 41$ : eGFP) control $(n=11)$ and LY294002-treated (5 $\mu \mathrm{M}, 30-60$ hpf) $(n=19)$ embryos were imaged at $4 \mathrm{dpf}$. Anterior to the left; $2 \mu \mathrm{m}$ step size.

Representative embryos are shown and the number of embryos that showed this pattern/total number of embryos is indicated. The number of GFP $^{\text {low }}$ HSPCs was determined at $50 \mathrm{hpf}(\mathbf{I})$ and $4 \mathrm{dpf}(\mathbf{m})$ and is expressed as average number of cells; error bars indicate standard error of the mean (SEM).

Shapiro-Wilk test for normal distribution and Welch's twotailed $t$-test were used for statistical analysis; $* * * p<$ 0.001 .

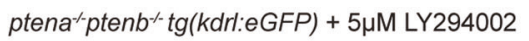
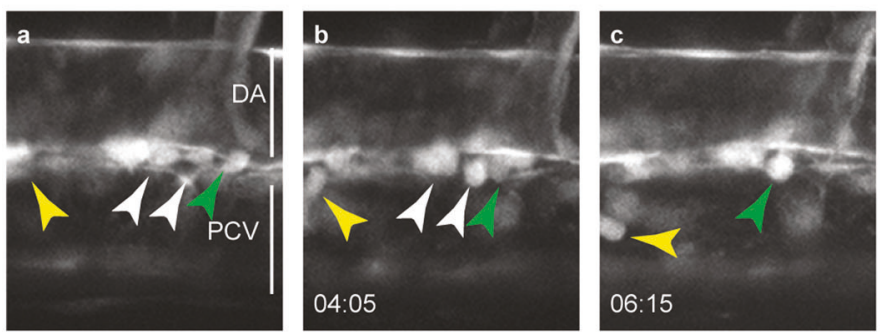

wildtype $\operatorname{tg}(k d r l: e G F P)+5 \mu \mathrm{M}$ LY294002
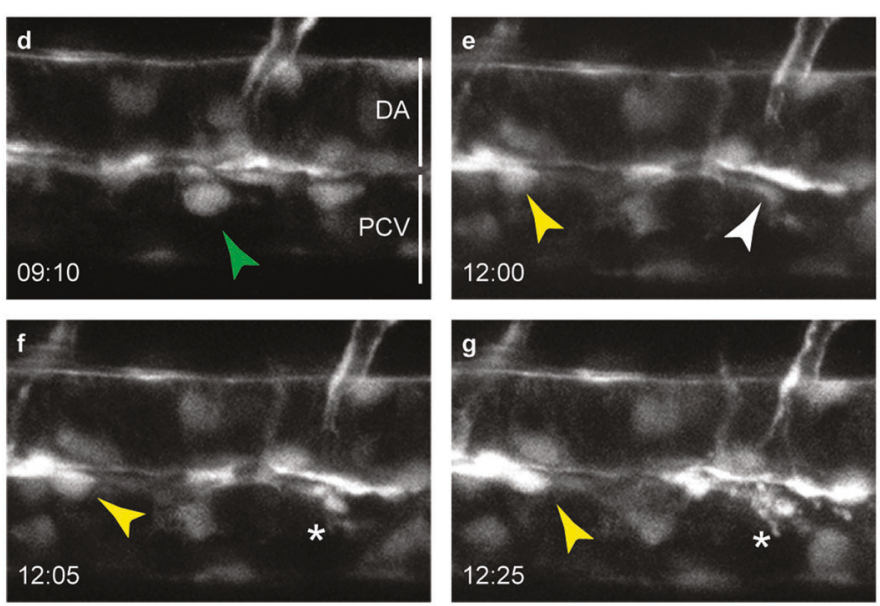

$\operatorname{tg}(\mathrm{kdrl}: m$ Cherry-CAAX) (cd41:eGFP) (50hpf)
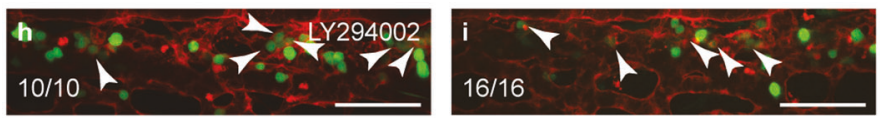

$\operatorname{tg}(c d 41: \mathrm{e} F F P) 4 \mathrm{dpf}$
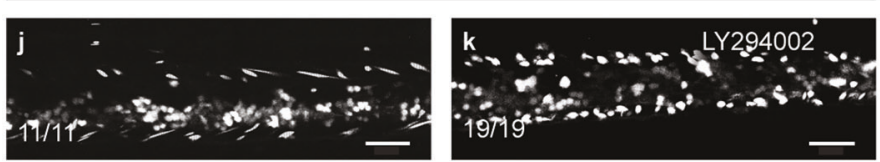

I
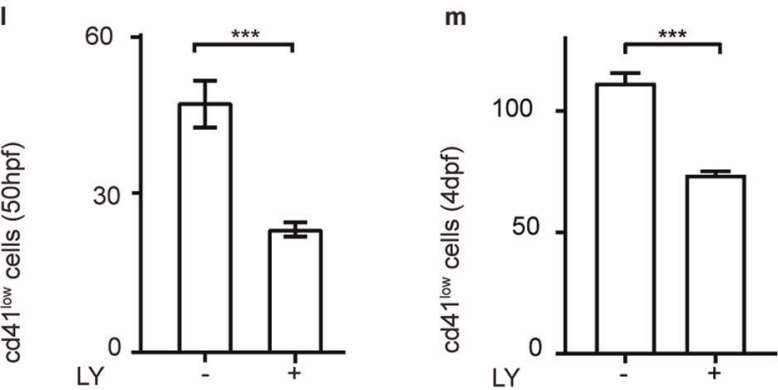

cells that were analyzed, 684 remained after filtering. RaceID3 separated the cells in distinct clusters (Fig. 6a). Cells in cluster 2 expressed erythrocyte progenitor-related genes (hbbe2, alas2, and cahz) (Fig. 6b). Cluster 3 is characterized by cells expressing genes related to thrombocyte/erythrocyte progenitors (gatala, klf1 [51, 55, 56]) (Fig. 6c). Cells in cluster 1 express genes indicative of
HSPCs, including $c-m y b$ (Fig. 6d). Cluster 4 represents early myeloid progenitors, as runx3, pu.l (also known as spilb), and cebpb [55] are highly expressed (Fig. 6e). Cluster 5 is characterized by neutrophil progenitor-related gene expression (mpx) (Fig. 6f). Analysis of the distribution of hematopoietic cells, using a Fisher's exact test indicated that the thrombocyte/erythrocyte progenitor cells were 

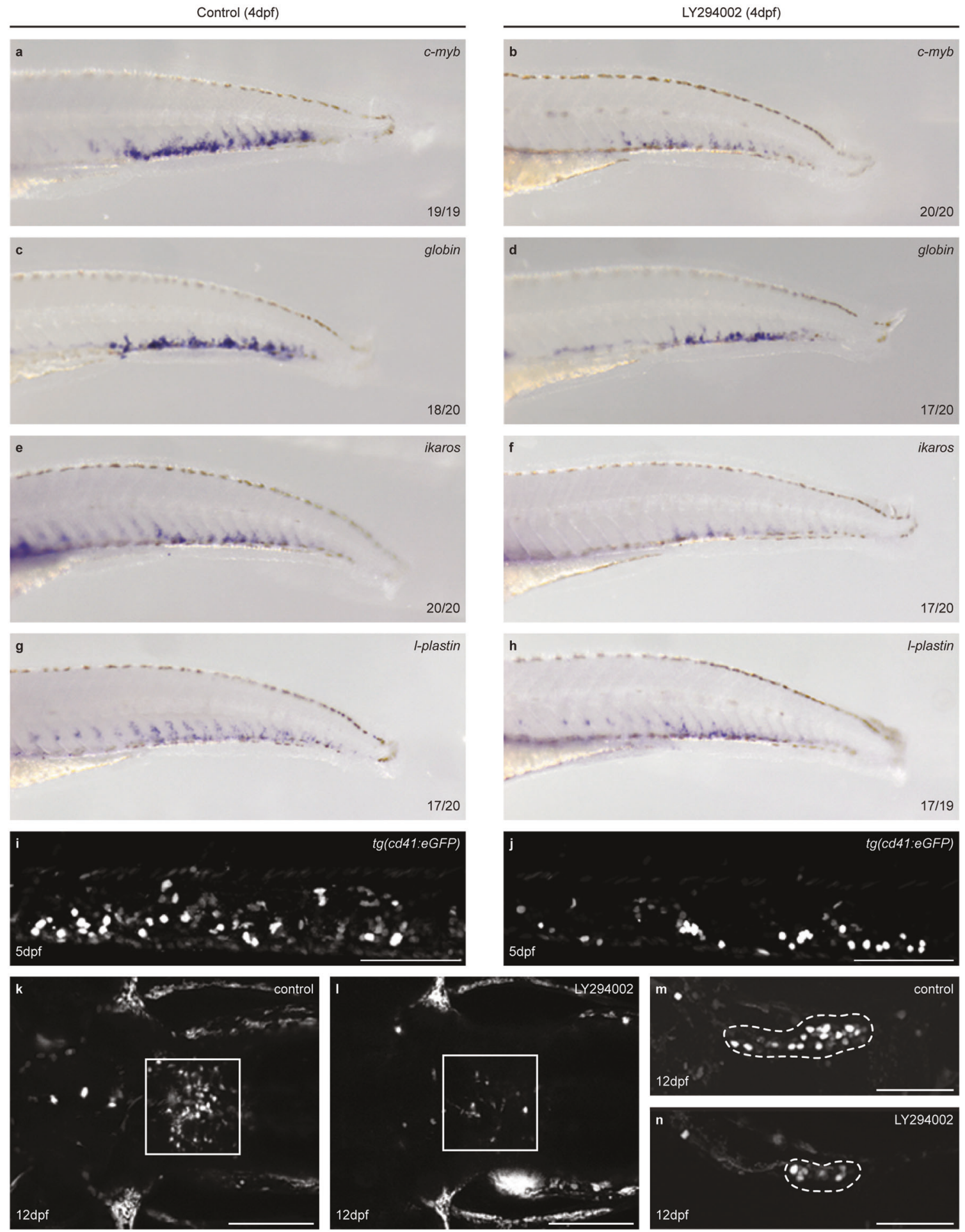

Fig. 4 HSPCs of LY294002-treated embryos engage in all blood lineages, but show impaired colonization of definitive hematopoietic organs. a-h Control and LY294002-treated (from 32-60 hpf) embryos were fixed at $4 \mathrm{dpf}$. Markers for definitive blood lineages were assessed by in situ hybridization in the CHT: c-myb (HSPCs; a, b), globin (erythrocyte lineage; c, d), ikaros (lymphocyte lineage; e, f), l-plastin (leukocytes; $\mathbf{g}, \mathbf{h}$ ). Representative embryos are shown, with anterior to the left. The number of embryos that showed a particular pattern/total number of embryos is indicated in the bottom right corner of each panel. i, j GFP ${ }^{\text {high }}$ thrombocytes were imaged at 5 dpf in $\operatorname{tg}(\mathrm{cd} 41$ :eGFP) embryos. Scale bar: $100 \mu \mathrm{m}$. k-n Highresolution imaging at $12 \mathrm{dpf}$ of kidney (k, l, dorsal view) (control, $n=6$; LY294002-treated, $n=8 ; 4 \mu \mathrm{m}$ step size) and thymus (m, $\mathbf{n}$, lateral view) (control, $n=6$; LY294002-treated, $n=7 ; 2 \mu \mathrm{m}$ step size). Anterior to the left; maximum projections of representative larvae. Scale bar: $100 \mu \mathrm{m}$. 
a

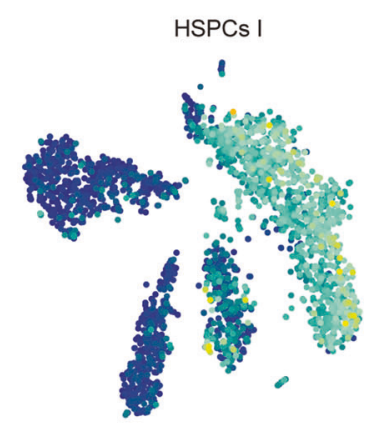

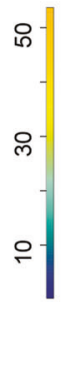

b

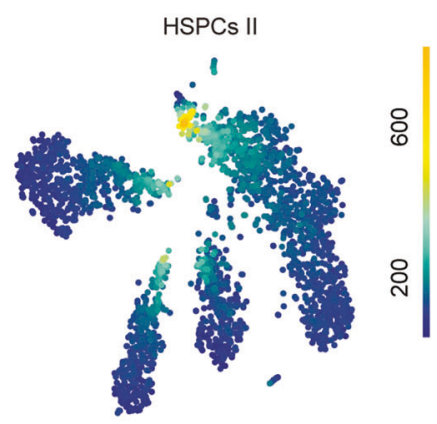

c

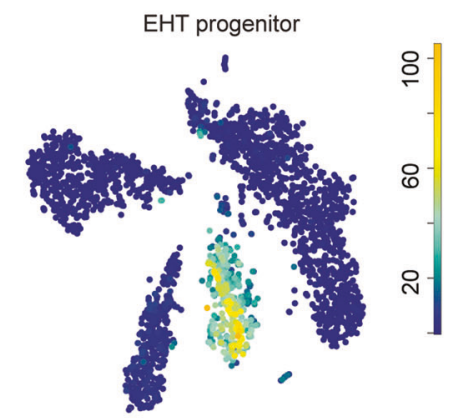

d

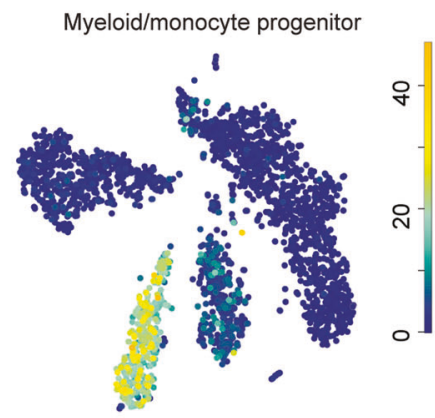

g

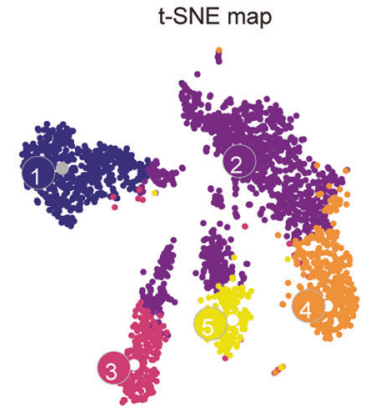

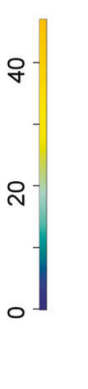

e

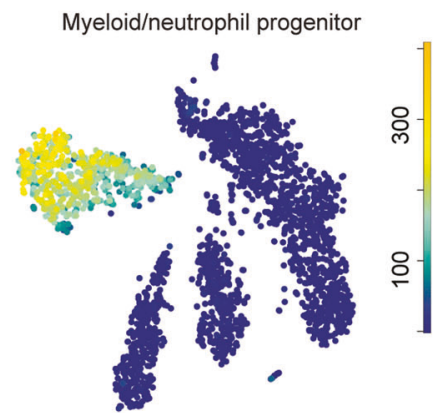

h
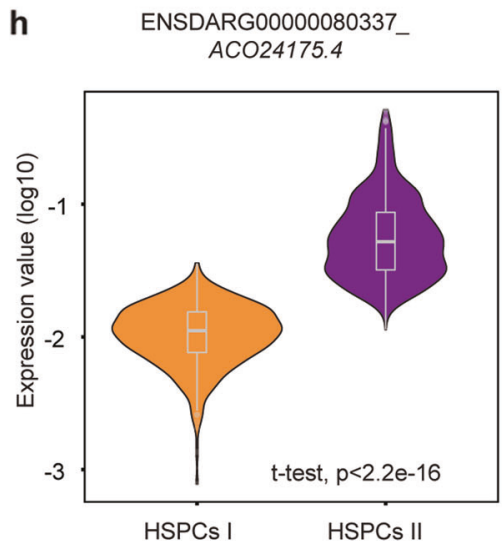

f

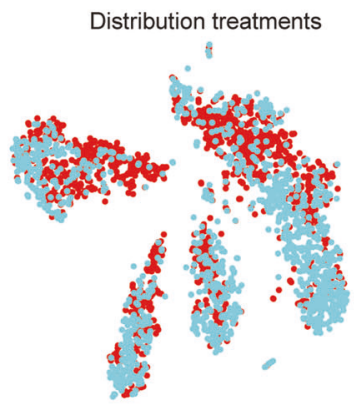

- LY294002

- wt

i

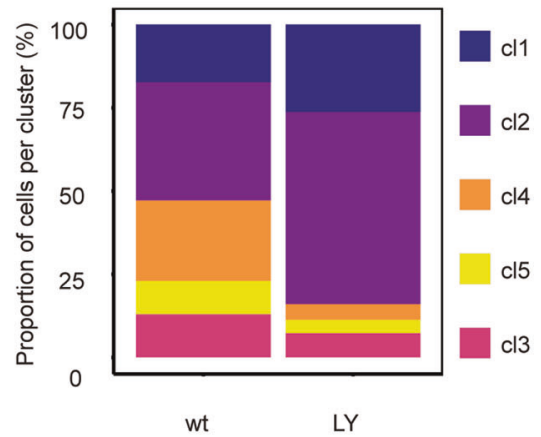

wt

dot represents a single cell. Colors and numbers indicate cluster and correspond to colors in $\mathbf{i}$. The distribution of in total 2512 cells over the five clusters are shown as percentage of total for control and LY294002-treated embryos. Fisher's exact test with multiple testing correction (Fdr) were used for statistical analysis. $* * * p<0.001$. cl1: Myeloid/neutrophil progenitor, cl2: HSPC II, cl3: myeloid/monocyte progenitor, cl4: HSPC I, cl5: EHT progenitor. h Normalized expression level of ENSDARG00000080337_ACO24175.4 for HSPC I and HSPC II cluster using violin plots. Normalized expression is plotted on a $\log 10$ scale. trophil progenitors. f t-SNE map highlighting the distribution of cells from LY294002-treated embryos and their controls (g). Visualization of k-medoid clustering and cell-to-cell distances using t-SNEs. Each

underrepresented in the LY294002-treated embryos $(p<$ $0.01)$ and HSPCs were significantly overrepresented $(p<$ 0.001) (Fig. 6g, h). These results indicate a significant shift toward HSPCs at the expense of the thrombocyte/ erythrocyte progenitor cluster in response to LY294002 treatment.

Likewise, we assessed transcriptomic differences by scRNA-seq in HSPCs from the CHT between ptena ${ }^{-/-}$ 


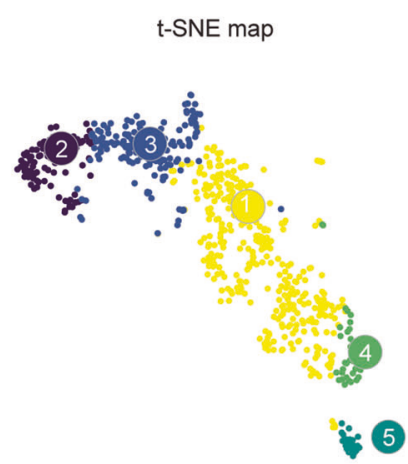

d

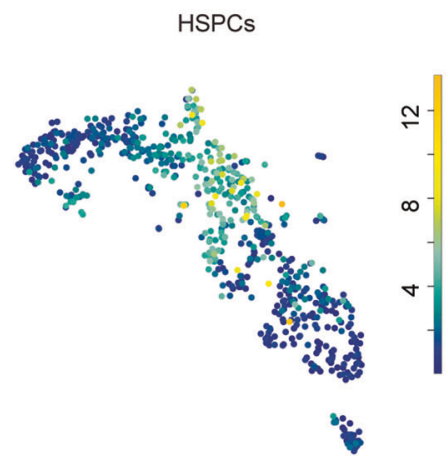

g

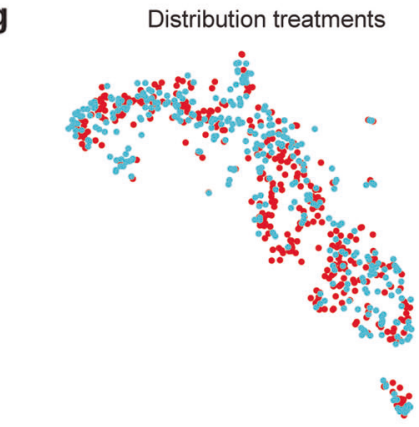

- LY294002

- wt

Fig. 6 ScRNA-seq seq reveals a shift toward HSPCs in LY294002treated 5 dpf old embryos. CHTs of control and LY294002-treated embryos (5 dpf, 100 embryos each) were dissected, pooled, dissociated, FACS sorted and submitted to SORT-seq. a Visualization of $\mathrm{k}$-medoid clustering and cell-to-cell distances using t-SNEs. Each dot represents a single cell. Colors and numbers indicate cluster and correspond to colors in $\mathbf{h}$. In total, 684 cells are shown. b-f t-SNEs maps highlighting the expression of marker genes for each of the different
C Thrombocyte/ erythrocyte progenitor

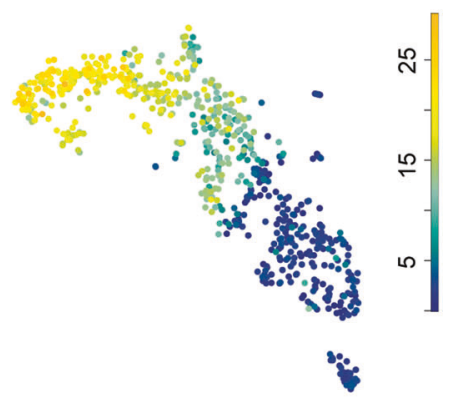

Neutrophil progenitor

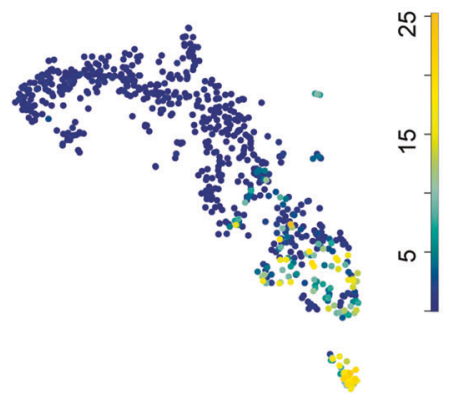

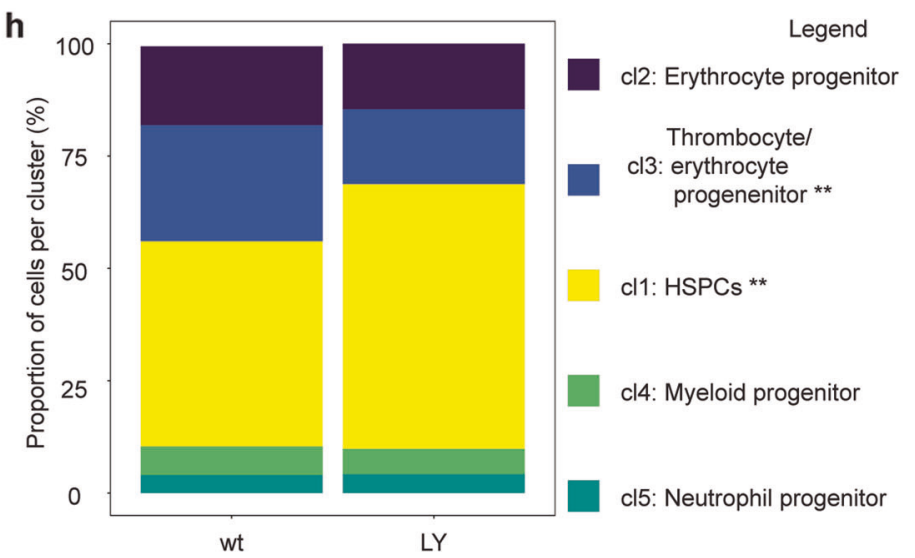

cell types found. Transcript counts are given in a linear scale. b Erythrocyte progenitors, c Thrombocyte/erythrocyte progenitors, d HSPCs, e Myeloid progenitors, f Neutrophil progenitors. g t-SNE map highlighting the distribution of LY294002-treated embryos and their controls (h). The percentage of cells from LY294002-treated embryos and their controls in the different clusters. Fisher's exact test with multiple testing correction (Fdr) were used for statistical analysis. $* * p<0.01, * * * p<0.001$.

pten $^{-/-}$mutant embryos and their siblings at $5 \mathrm{dpf}$. Approximately 100 ptena $^{-/-}$pten $^{-/-}$mutant embryos and siblings were selected based on phenotype [15], which yielded $614 \mathrm{~cd} 41^{\text {low }}$ cells after filtering. RaceID3 indicated that clusters emerged representing the same hematopoietic lineages as described for the wild-type and LY294002treated data (cf. Figs. 6 and 7). Analysis of the distribution of hematopoietic cells from pten mutants and their siblings over the five clusters indicated that the erythrocyte- and neutrophil progenitor cells were overrepresented in the pten 
a

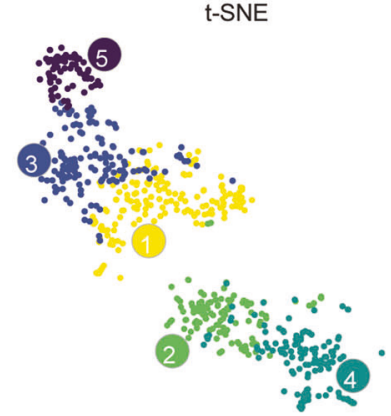

d
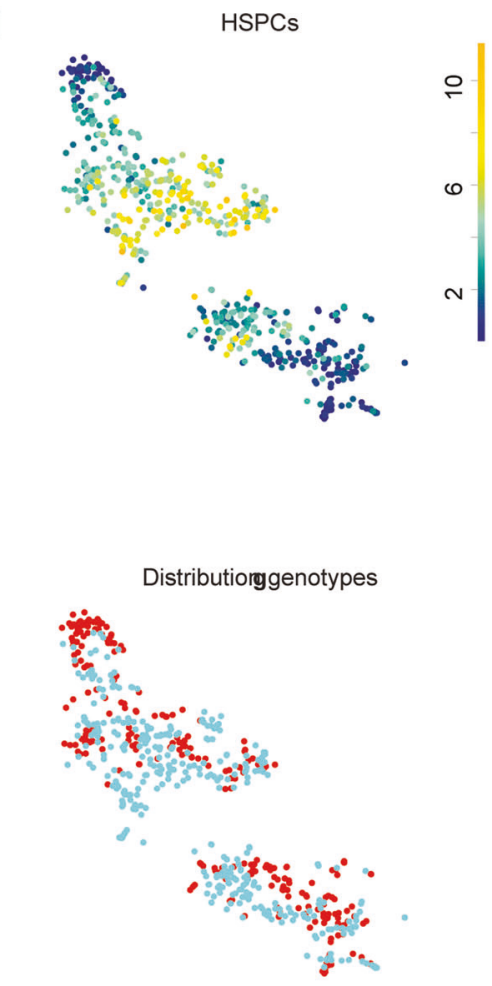

- Mutant

- Sibling b

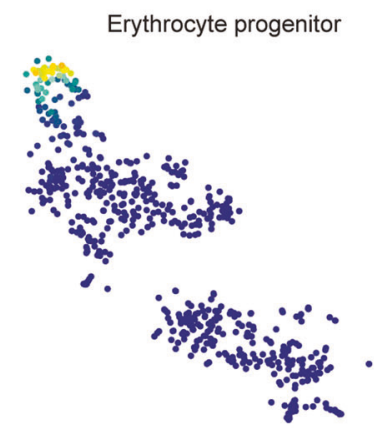

C

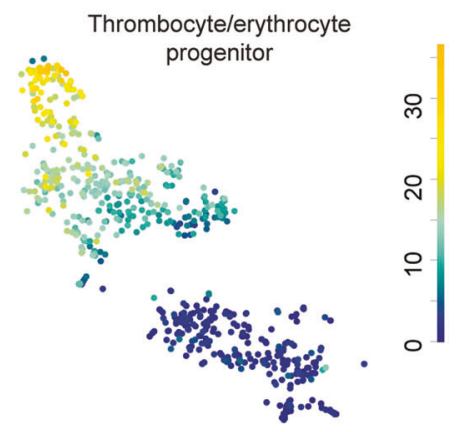

e
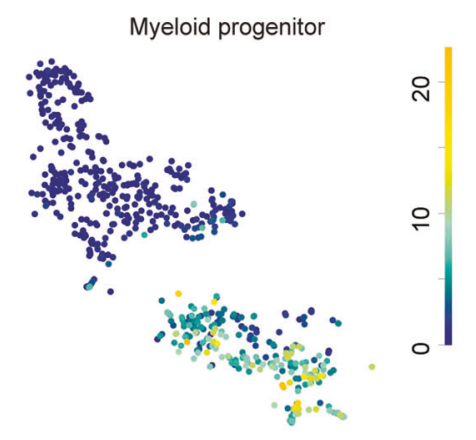

f

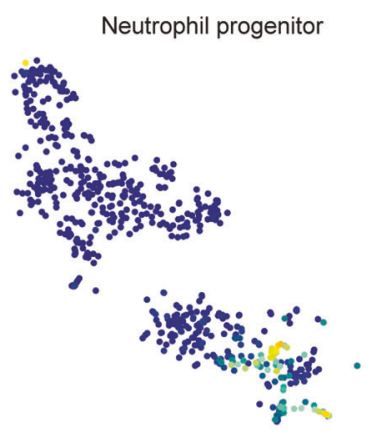

이
0
0
Fig. 7 ScRNA-seq reveals a shift toward more differentiated cell types in $5 \mathrm{dpf}$ old ptena $^{-/-}$ptenb $^{-/-}$mutant embryos. CHTs of control and ptena ${ }^{-/}$pten $^{-/-}$mutant embryos (5 dpf, $\sim 100$ embryos each) were dissected, pooled, dissociated, FACS sorted and submitted to SORT-seq. a Visualization of k-medoid clustering and cell-to-cells distances using t-SNEs. Each dot represents a single cell. Colors and numbers indicate cluster and correspond to colors in $\mathbf{h}$. In total, 614 cells are shown. b-f t-SNEs maps highlighting the expression of marker genes for each of the different cell types found. Transcript

mutant $(p<0.001$ and $p<0.05)$ and that HSPCs were significantly underrepresented $(p<0.001)$ (Figs. $7 \mathrm{~g}$, h and S6). These results indicate a significant shift in ptena ${ }^{-/}$pten $^{-/-}$ mutant embryos toward erythrocyte progenitor and neutrophil progenitors at the expense of HSPCs.

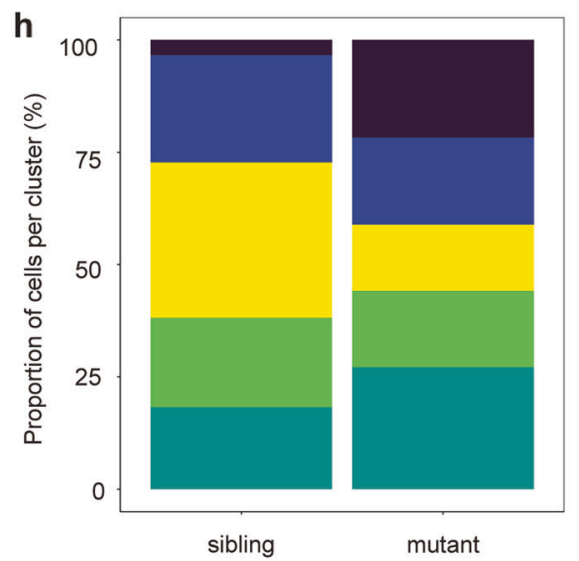

counts are given in a linear scale. b Erythrocyte progenitors, c Thrombocyte/erythrocyte progenitor, d HSPCs, e Myeloid progenitors, f Neutrophil progenitors. g t-SNE map highlighting the distribution of pten $^{-/-}$ptenb ${ }^{-/-}$mutant embryos and their siblings. $\mathbf{h}$ The percentages of cells from ptena $^{-/-}$pten $b^{-/-}$mutant embryos and their siblings in the different clusters. Fisher's exact test with multiple testing correction $(\mathrm{Fdr})$ were used for statistical analysis. ${ }^{*} p<0.05$, $* * * p<0.001$.

\section{Discussion}

We used zebrafish mutant embryos lacking functional Pten to investigate how loss of Pten affects the ontogeny of hematopoiesis. Characterization of zebrafish ptena $^{-/-}$ 
pten $^{-/-}$mutant embryos led to the unexpected finding that half of the HSPCs undergo apoptosis upon emergence from the VDA during EHT at the onset of the definitive wave (Fig. 1). Loss of function of Pten is usually linked to enhanced cell survival, such as for instance in Pten knockout mice [57]. We reported that $\mathrm{y}$-irradiation reduces apoptosis in ptena ${ }^{-/-}$ptenb $b^{-/-}$mutant embryos [15]. Apoptosis of zebrafish HSPCs has been reported before, in that grechetto mutants display decreasing numbers of HSPCs due to apoptosis [52]. Runx1 knockdown also induced abortive EHT events due to apotosis [5]. Runxl expression was not affected in the VDA of ptena ${ }^{-/-}$ pten $^{-/-}$mutants (Fig. S1), suggesting that the mechanism underlying EHT defects in ptena ${ }^{-/-}$ptenb ${ }^{-/-}$mutant embryos and Runx1 morphants are distinct. Apoptosis of HSPCs in pten mutants is due to enhanced PI3K-mediated signaling, because treatment with a PI3K inhibitor rescued apoptosis of HSPCs. Surprisingly, treatment of wild-type embryos with the PI3K inhibitor induced death of half of the HSPCs upon emergence from the VDA as well (Fig. 3). These results suggest that upon emergence from the VDA, HSPCs require a moderate level of PI3K signaling, as hyperactivation of $\mathrm{PI} 3 \mathrm{~K}$ signaling in Pten mutants as well as inhibition of PI3K signaling induced apoptosis of emerging HSPCs.

After emerging from the VDA, the surviving HSPCs enter circulation and seed the CHT, as demonstrated by photoconversion of endothelial cells prior to EHT in ptena $^{-/}$pten $^{-/-}$mutant embryos and siblings (Fig. 2). Half of the HSPCs of ptena ${ }^{-/-}$ptenb ${ }^{-/-}$mutant embryos and LY294002-treated embryos colonized the CHT, compared to wild-type embryos (Figs. 2 and 3 and Table 1). In LY294002-treated embryos, the decrease in HSPCs remained, whereas in ptena ${ }^{-/}$pten $b^{-/-}$mutant embryos the surviving HSPCs hyperproliferate leading to an increase in HSPCs at later stages [17]. Surviving HSPCs from ptena $^{-/-}$pten $b^{-/-}$mutants engage in all blood lineages [17]. However, definitive differentiation of major blood lineages is arrested in the ptena ${ }^{-/}$pten $b^{-/-}$mutants, consistent with the inverse correlation of proliferation and differentiation of stem cells [58]. The surviving HSPCs of LY294002-treated embryos also engaged in all blood lineages (Fig. 4), demonstrating pluripotency of the HSPCs.

Using scRNA-seq at the onset of the definitive wave (36hpf) of hematopoiesis two HSPC clusters were identified, that both expressed HSPC markers. In control embryos equal numbers of cells populated the HSPCs I and HSPCs II clusters. Predominantly the cells from the HSPCs I cluster were lost upon PI3K inhibition (Fig. 5). Our imaging data indicated that half of the HSPCs disintegrated upon treatment with LY294002 (Fig. 3). It is tempting to speculate that the surviving half of the HSPCs all belong to the HSPCs II cluster. Whereas both HSPCs clusters expressed
HSPC markers, several genes are significantly differentially expressed (Fig. S4), albeit subtle. Expression of ENSDARG00000080337_ACO24175.4 and to a lesser extent tmed $1 b$ distinguished the HSPCs II cluster from the HSPCs I cluster. In situ hybridization using an ENSDARG00000080337_ACO24175.4-specific probe indicated high expression throughout the embryo, which did not allow validation of the difference in expression in HSPCs I and HSPCs II cells (Fig. S3). Little is known about ENSDARG00000080337_ACO24175.4, except that it is a mitochondrial ribosomal gene (mt rDNA). Interestingly, HSCs have significantly lower rates of protein synthesis than other hematopoietic cells [59]. The protein product of ENSDARG00000080337_ACO24175.4 may have a role in protein synthesis. Hence, the difference in expression levels may indicate that the HSPCs II cells that survive PI3K inhibition are less stem cell-like and more progenitor-like, poised to differentiate.

In response to LY294002 treatment, the number of cd $41^{\text {low }}$ HSPCs was reduced in the CHT at $4 \mathrm{dpf}$ and in the definitive hematopoietic organs at 8 and 12 dpf (Figs. 3 and 4). scRNA-seq of putative HSPCs (cd41 low, $\mathrm{kdrl}^{+}$cells) at the end of the definitive wave $(5 \mathrm{dpf})$ indicated initiation of differentiation in different blood lineages (Fig. 6), consistent with in situ hybridization (Fig. 4). Yet, inhibition of PI3K arrested differentiation, i.e. increased HSPC fate, predominantly at the expense of thrombocyte/erythrocyte progenitor fate (Fig. 6). Overall, it is evident that there is a significant reduction in hematopoietic cell number (Figs. 4 and 6), which may be caused by preferential loss of HSPCs with more stem cell-like properties (Fig. 5).

ScRNA-seq at the end of the definitive wave showed a significant increase in erythrocyte- and neutrophil progenitors in ptena $^{-/-}$ptenb $^{-/-}$mutant embryos (Figs. 7 and S6), consistent with earlier in vivo data [17]. However, we reported an overall increase in HSPCs, due to hyperproliferation, whereas here, we observed a decrease in HSPCs in the scRNA-seq data. An explanation for this apparent discrepancy is that the hyperproliferating HPSCs we observed earlier [17] actually have initiated differentiation already and are scored as erythrocyte and neutrophil progenitors by scRNA-seq.

Conditional knock-out of Pten in HSCs in mouse adult bone marrow drives HSCs into the cell cycle, resulting in transient expansion of the spleen and eventually in depletion of HSCs in the bone marrow. These conditional PTENdeficient mice die of a myeloproliferative disorder that resembles acute myeloid/lymphoid leukemia, indicating that PTEN is required for maintenance of HSCs [13, 14]. It is noteworthy that there are differences between the conditional mouse models and the zebrafish model we used. In the mouse, Pten is deleted in adult bone marrow cells, well after HSCs have formed, whereas in zebrafish, Pten is 
systemically deleted and therefore effective prior to the emergence of HSPCs. Studies in mice showed that regardless of cell state, HSCs and multipotent progenitors had a lower protein synthesis rate than more restricted hematopoietic progenitors. Loss of PTEN in HSCs caused depletion of HSCs, due to a higher rate of protein synthesis [59], which is consistent with our observation that loss of Pten in zebrafish caused HSPCs to hyperproliferate and become less stem cell like.

Long-term HSCs are quiescent, whereas short-term HSCs proliferate more [2]. It would be tempting to speculate that the HSPCs that undergo apoptosis upon loss of Pten or upon $\mathrm{PI} 3 \mathrm{~K}$ inhibition are involved in long-term colonization of definitive hematopoietic organs. The surviving HSPCs in pten mutants at the onset of the definitive wave would then represent multipotent progenitors that only have limited potential for self-renewal. Investigating the regulatory network underlying the surviving and disintegrating HSPCs will further expand our understanding of short- and longlived HSPCs and will eventually contribute to the development of efficient stem cell-based therapies [60, 61].

\section{Methods}

\section{Zebrafish husbandry}

Ptena $^{-/-}$ptenb $^{-/-}$, ptena $^{-/-}$ptenb $^{-/-}$[15], Tg(kdrl:eGFP $)$ [18], $T g(k d r l: m C h e r r y-C A A X)$ [19], and $T g(c d 41: e G F P)$ [20] were maintained according to FELASA guidelines, crossed, raised, and staged as described [21-23]. Pten mutant fish (embryos) were genotyped by sequencing [15]. The $t g(k d r l: D e n d r a 2)$ line was derived by Tol2-mediated transgenesis [24] of a construct containing the $\sim 7.0 \mathrm{~kb} \mathrm{kdrl}$ promoter (a kind gift from D. Stainier), driving the expression of Dendra2 [25]. From 24hpf onwards, all embryos were grown in PTU-containing medium to block pigmentation.

\section{LY294002 treatment}

Embryos were treated with $5 \mu \mathrm{M}$ LY294002 (Calbiochem, San Diego, CA, USA) or DMSO control in the dark. For early treatment, embryos were incubated with LY294002 from $32 \mathrm{hpf}$ onwards and mounted after $4 \mathrm{~h}$ for time-lapse confocal imaging. For late treatment and to investigate thymus and kidney colonization, embryos were treated with $5 \mu \mathrm{M}$ LY294002 from 32 to $60 \mathrm{hpf}$ and imaged.

\section{Constructs, mRNA synthesis, and microinjections}

The Ptenb-mCherry fusion construct in the vector pCS2+ was obtained as described in refs. $[15,26]$ and linearized with NotI. To synthesize $5^{\prime}$ capped sense mRNA, the
mMessage mMachine SP6 kit (Ambion) was used. mRNA injections were performed at the one-cell stage using a total of $300 \mathrm{pg}$ of mRNA.

\section{Confocal, fluorescence, brightfield microscopy, and time-lapse imaging}

Fluorescence images of transgenic embryos were acquired using TCS-SPE and time-lapse imaging using TCS-SP2 as described [27] and processed with ImageJ [28]. For all live imaging embryos were anesthetized with tricaine [21], mounted on a glass cover dish with $0.7 \%$ low melting agarose and covered with standard E3 medium. Whole mount bright field images were taken with a Leica DC 300F stereomicroscope.

\section{In situ hybridization}

Whole mount in situ hybridization was performed according to standard protocols [29] and images were taken using a Zeiss Axioplan microscope connected to a Leica DFC480 camera.

\section{Acridine orange staining and whole mount immunohistochemistry}

Embryos were incubated with $5 \mu \mathrm{g} / \mathrm{ml}$ acridine orange [30] for 20 min between 35 and $40 \mathrm{hpf}$ and subsequently washed with standard E3 medium. Embryos were then imaged as described above. Immunohistochemical labeling performed using fixed (40 hpf) embryos to detect apoptosis using an activated caspase-3-specific antibody (BD Pharmingen) [31]. After confocal images were collected embryos were genotyped.

\section{Photoconversion}

Fluorescent tracing of VDA-derived HSPCs colonizing the CHT was done using the $t g(k d r l: D e n d r a 2)$ line as described before $[32,33]$ with a Leica SP5 confocal microscope with a $\times 20$ dry objective. At $28 \mathrm{hpf}$ an area of $\sim 40 \times 750 \mathrm{~nm}$ around the VDA, parallel to the yolk sac extension was photoconverted. The $405 \mathrm{~nm}$ UV laser intensity and exposure time were optimized for strong Dendra2-conversion without cell damage. After photoconversion embryos were transferred to E3 medium and at 50-60 hpf their CHT areas were imaged on a Leica SPE Live confocal microscope using a $\times 20$ dry objective. To exclude bleed-through of Dendra-green, red channel detection was set stringently $(630-680 \mathrm{~nm})$.

\section{Quantification of GFPlow progenitor cells using $t g$ (cd41:eGFP)}

$\mathrm{GFP}^{\text {low }}$ and GFP ${ }^{\text {high }}$ expressing cells were quantified in the $\mathrm{CHT}$ at $48 \mathrm{hpf}, 50 \mathrm{hpf}$ or $4 \mathrm{dpf}$ using confocal imaging and 
Volocity and Imaris software. Ptena ${ }^{+/-}$pten $^{-/-}$mutants on $\operatorname{tg}(c d 41: e G F P)$ background were crossed and offspring was mounted at 48 hpf. Wild-type $\operatorname{tg}(c d 41: e G F P)$ embryos were treated with $5 \mu \mathrm{M}$ LY294002 as described above and mounted and imaged at $50 \mathrm{hpf}$ or $4 \mathrm{dpf}$. All GFP Gow $^{\text {low }}$ expressing cells were counted in the entire CHT.

\section{Flow cytometry}

The aorta-gonad-mesonephros (AGM) of $~ 400036 \mathrm{hpf}$ and 400 CHTs of $5 \mathrm{dpf}$ old $t g(k d r l: m$ Cherry/cd41:eGFP) embryos were dissected and collected in Leibovitz medium. After washing with phosphate-buffered saline, the AGMs were deyolked using calcium-free Ringer's solution $(116 \mathrm{mM} \mathrm{NaCl}, 2.9 \mathrm{mM} \mathrm{KCl}$ and $5 \mathrm{mM}$ HEPES) and then AGMs and CHTs were dissociated in TrypLE Express (Gibco) for $45 \mathrm{~min}$ at $32^{\circ} \mathrm{C}$. The resulting cell suspension was washed in phosphate-buffered saline and passed through a $40-\mu \mathrm{m}$ filter after resuspension in phosphatebuffered saline supplemented with $2 \mathrm{mM}$ ethylenediaminetetraacetic acid, $2 \%$ fetal calf serum, and $0.5 \mu \mathrm{g} / \mathrm{ml} \mathrm{4^{ \prime } , 6 -}$ diamidino-2-phenylindole (DAPI), to exclude dead cells. Cells with kdrl- and cd4 $41^{\text {low }}$-positive signal were subjected to fluorescence-activated cell sorting (FACS) using a BD FACSAriaII and BD FACSFusion.

\section{ScRNA-seq with SORT-seq}

ScRNA-seq was performed by Single-Cell Discoveries BV (Utrecht, the Netherlands), according to an adapted version of the SORT-seq protocol [34, 35], with adapted primers described in ref. [36].

\section{Data analysis}

During sequencing, Read1 used for identification of the Ilumina library barcode, cell barcode, and UMI. Read2 was used to map to the reference transcriptome of Zv9 Danio rerio. Data were demultiplexed as described [37]. Single-cell transcriptomics analysis was done using the RaceID3 algorithm, following an adapted version of the RaceID manual (https://cran.r-project.org/web/packages/RaceID/vignettes/

RaceID.html). Cells that had $<1500$ UMIs and genes that were detected in $<5$ UMIs in five cells were discarded. The number of initial clusters was set at 5. Differential gene expression analysis was done as described in ref. [34] with an adapted version of the DESseq2 algorithm [38].

\section{Data availability}

For original data, please contact j.denhertog@ hubrecht.eu. scRNA data are available at GEO under accession number GSE166900.
Acknowledgements The authors would like to thank Mark Reijnen and animal caretakers for excellent management of the fish facility. Microscopy was done in the Hubrecht Imaging Centre. The authors would like to thank Stefan van der Elst and Reinier van der Linden for FACS-sorting. The authors would like to thank Jeroen Paardekooper Overman for statistical analysis, Laila Ritsma, Sylvain de Rossi, and Miriam Stumpf for technical support and Bas Castelijns for help with scRNA data analysis. This work was supported in part by an EU (FP7) grant, ZF-CANCER (HEALTH-F2-2008-201439).

Author contributions SB-F, SC, and JdH designed experiments with input from KK and PH; SB-F and SC performed the experiments; BP and SSM generated the $\operatorname{Tg}(\mathrm{kdrl}:$ Dendra $)$ line and helped perform the photoconversion experiments; $\mathrm{KK}$ and $\mathrm{JdH}$ supervised the work; SB-F, SC, PH, KK, and JdH wrote the manuscript.

\section{Compliance with ethical standards}

Conflict of interest The authors declare no competing interests.

Ethical approval All animal experiments described in this manuscript were approved by the local animal experiments committee (Hubrecht Institute: Koninklijke Nederlandse Akademie van WetenschappenDierexperimenten commissie protocol HI180701 and University Montpellier: Direction Sanitaire et Vétérinaire de l'Hérault and Comité d'Ethique pour l'Expérimentation Animale under reference CEEALR-13007) and performed according to local guidelines and policies in compliance with national and European law.

Publisher's note Springer Nature remains neutral with regard to jurisdictional claims in published maps and institutional affiliations.

Open Access This article is licensed under a Creative Commons Attribution 4.0 International License, which permits use, sharing, adaptation, distribution and reproduction in any medium or format, as long as you give appropriate credit to the original author(s) and the source, provide a link to the Creative Commons license, and indicate if changes were made. The images or other third party material in this article are included in the article's Creative Commons license, unless indicated otherwise in a credit line to the material. If material is not included in the article's Creative Commons license and your intended use is not permitted by statutory regulation or exceeds the permitted use, you will need to obtain permission directly from the copyright holder. To view a copy of this license, visit http://creativecommons. org/licenses/by/4.0/.

\section{References}

1. Kondo M, Wagers AJ, Manz MG, Prohaska SS, Scherer DC, Beilhack GF, et al. Biology of hematopoietic stem cells and progenitors: implication for clinical application. Annu Rev Immunol. 2003;21:759-806.

2. Orkin SH, Zon LI. Hematopoiesis: an evolving paradigm for stem cell biology. Cell. 2008;132:631-44.

3. Stachura DL, Traver D. Cellular dissection of zebrafish hematopoiesis. Methods Cell Biol. 2011;101:75-110.

4. Bertrand JY, Chi NC, Santoso B, Teng S, Stainier DYR, Traver D. Haematopoietic stem cells derive directly from aortic endothelium during development. Nature. 2010;464:108-11.

5. Kissa K, Herbomel P. Blood stem cells emerge from aortic endothelium by a novel type of cell transition. Nature. 2010;464:112-5.

6. Boisset JC, van Cappellen W, Andrieu-Soler C, Galjart N, Dzierzak E, Robin C. In vivo imaging of haematopoietic cells 
emerging from the mouse aortic endothelium. Nature. 2010;464: 116-20.

7. Godin I, Cumano A. The hare and the tortoise: an embryonic haematopoietic race. Nat Rev Immunol. 2002;2:593-604.

8. Murayama E, Kissa K, Zapata A, Mordelet E, Briolat V, Lin HF, et al. Tracing hematopoietic precursor migration to successive hematopoietic organs during zebrafish development. Immunity. 2006;25:963-75.

9. Palomero T, Sulis ML, Cortina M, Real PJ, Barnes K, Ciofani M, et al. Mutational loss of PTEN induces resistance to NOTCH1 inhibition in T-cell leukemia. Nat Med. 2007;13:1203-10.

10. Van Vlierberghe P, Ferrando A.The molecular basis of $T$ cell acute lymphoblastic leukemia. J Clin Invest. 2012;122:3398-406.

11. Song MS, Salmena L, Pandolfi PP. The functions and regulation of the PTEN tumour suppressor. Nat Rev Mol Cell Biol. 2012;13:283-96.

12. Blackburn JS, Liu S, Wilder JL, Dobrinski KP, Lobbardi R, Moore FE, et al. Clonal evolution enhances leukemiapropagating cell frequency in $\mathrm{T}$ cell acute lymphoblastic leukemia through Akt/mTORC1 pathway activation. Cancer Cell. 2014;25:366-78

13. Yilmaz ÖH, Valdez R, Theisen BK, Guo W, Ferguson DO, Wu H, et al. Pten dependence distinguishes haematopoietic stem cells from leukaemia-initiating cells. Nature. 2006;441:475-82.

14. Zhang J, Grindley JC, Yin T, Jayasinghe S, He XC, Ross JT, et al. PTEN maintains haematopoietic stem cells and acts in lineage choice and leukaemia prevention. Nature. 2006;441: 518-22.

15. Faucherre A, Taylor GS, Overvoorde J, Dixon JE, den Hertog J. Zebrafish pten genes have overlapping and non-redundant functions in tumorigenesis and embryonic development. Oncogene. 2008;27:1079-86.

16. Choorapoikayil S, Kuiper RV, De Bruin A, den Hertog J. Haploinsufficiency of the genes encoding the tumor suppressor Pten predisposes zebrafish to hemangiosarcoma. Dis Model Mech. 2012;5:241-7.

17. Choorapoikayil S, Kers R, Herbomel P, Kissa K, den Hertog J. Pivotal role of Pten in the balance between proliferation and differentiation of hematopoietic stem cells in zebrafish. Blood. 2014;123:184-90.

18. Jin S-W, Beis D, Mitchell T, Chen J-N, Stainier DYR. Cellular and molecular analyses of vascular tube and lumen formation in zebrafish. Development. 2005;132:5199-209.

19. Hogan BM, Bos FL, Bussmann J, Witte M, Chi NC, Duckers HJ, et al. Ccbe1 is required for embryonic lymphangiogenesis and venous sprouting. Nat Genet. 2009;41:396-8.

20. Lin HF, Traver D, Zhu H, Dooley K, Paw BH, Zon LI, et al. Analysis of thrombocyte development in CD41-GFP transgenic zebrafish. Blood. 2005;106:3803-10.

21. Westerfield M. The zebrafish book: a guide for the laboratory use of zebrafish (Danio rerio). 4th ed. Eugene: University of Oregon Press; 2000

22. Kimmel CB, Ballard WW, Kimmel SR, Ullmann B, Schilling TF. Stages of embryonic development of the zebrafish. Dev Dyn. 1995;203:253-310.

23. Aleström P, D’Angelo L, Midtlyng PJ, Schorderet DF, SchulteMerker S, Sohm F, et al. Zebrafish: housing and husbandry recommendations. Lab Anim. 2020;54:213-24.

24. Urasaki A, Morvan G, Kawakami K. Functional dissection of the Tol2 transposable element identified the minimal cis-sequence and a highly repetitive sequence in the subterminal region essential for transposition. Genetics. 2006;174:639-49.

25. Gurskaya NG, Verkhusha VV, Shcheglov AS, Staroverov DB, Chepurnykh TV, Fradkov AF, et al. Engineering of a monomeric green-to-red photoactivatable fluorescent protein induced by blue light. Nat Biotechnol. 2006;24:461-5.
26. Stumpf M, Blokzijl-Franke S, den Hertog J. Fine-tuning of Pten localization and phosphatase activity is essential for zebrafish angiogenesis. PLoS ONE. 2016;11:e0154771.

27. Renaud O, Herbomel P, Kissa K. Studying cell behavior in whole zebrafish embryos by confocal live imaging: application to hematopoietic stem cells. Nat Protoc. 2011;6:1897-904.

28. Schneider CA, Rasband WS, Eliceiri KW. NIH Image to ImageJ: 25 years of image analysis. Nat Methods. 2012;9:671-5.

29. Thisse C, Thisse B. High-resolution in situ hybridization to whole-mount zebrafish embryos. Nat Protoc. 2008;3:59-69.

30. Darzynkiewicz Z, Bruno S, Del Bino G, Gorczyca W, Hotz MA, Lassota $\mathrm{P}$, et al. Features of apoptotic cells measured by flow cytometry. Cytometry. 1992;13:795-808.

31. Bolli N, Payne EM, Rhodes J, Gjini E, Johnston AB, Guo F, et al. Cpsf1 is required for definitive HSC survival in zebrafish. Blood. 2011;117:3996-4007.

32. Dixon G, Elks PM, Loynes CA, Whyte MKB, Renshaw SA. A method for the in vivo measurement of zebrafish tissue neutrophil lifespan. ISRN Hematol. 2012;2012:915868.

33. Tian Y, Xu J, Feng S, He S, Zhao S, Zhu L, et al. The first wave of $\mathrm{T}$ lymphopoiesis in zebrafish arises from aorta endothelium independent of hematopoietic stem cells. J Exp Med. 2017;214:3347-60.

34. Muraro MJ, Dharmadhikari G, Grün D, Groen N, Dielen T, Jansen E, et al. A single-cell transcriptome atlas of the human pancreas. Cell Syst. 2016;3:385-94.

35. Hashimshony T, Senderovich N, Avital G, Klochendler A, de Leeuw Y, Anavy L, et al. CEL-Seq2: sensitive highly-multiplexed single-cell RNA-Seq. Genome Biol. 2016;17:77.

36. Van Den Brink SC, Sage F, Vértesy Á, Spanjaard B, PetersonMaduro J, Baron CS, et al. Single-cell sequencing reveals dissociation-induced gene expression in tissue subpopulations. Nat Methods. 2017;14:935-6.

37. Grün D, Kester L, van Oudenaarden A. Validation of noise models for single-cell transcriptomics. Nat Methods. 2014;11:637-40.

38. Love MI, Huber W, Anders S. Moderated estimation of fold change and dispersion for RNA-seq data with DESeq2. Genome Biol. 2014;15:550.

39. Gering M, Patient R. Hedgehog signaling is required for adult blood stem cell formation in zebrafish embryos. Dev Cell. 2005;8:389-400.

40. Choorapoikayil S, Weijts B, Kers R, de Bruin A, den Hertog J. Loss of Pten promotes angiogenesis and enhanced vegfaa expression in zebrafish. Dis Model Mech. 2013;6:1159-66.

41. Bertrand JY, Giroux S, Golub R, Klaine M, Jalil A, Boucontet L, et al. Characterization of purified intraembryonic hematopoietic stem cells as a tool to define their site of origin. Proc Natl Acad Sci USA. 2005;102:134-9.

42. Kissa K, Murayama E, Zapata A, Cortés A, Perret E, Machu C, et al. Live imaging of emerging hematopoietic stem cells and early thymus colonization. Blood. 2008;111:1147-56.

43. Herman JS, Sagar, Grün D. FateID infers cell fate bias in multipotent progenitors from single-cell RNA-seq data. Nat Methods. 2018;15:379-86.

44. Baron CS, Kester L, Klaus A, Boisset JC, Thambyrajah R, Yvernogeau L, et al. Single-cell transcriptomics reveal the dynamic of haematopoietic stem cell production in the aorta. Nat Commun. 2018;9:2517.

45. Kowalczyk MS, Tirosh I, Heckl D, Rao TN, Dixit A, Haas BJ, et al. Single-cell RNA-seq reveals changes in cell cycle and differentiation programs upon aging of hematopoietic stem cells. Genome Res. 2015;25:1860-72.

46. Nestorowa S, Hamey FK, Sala BP, Diamanti E, Shepherd M, Laurenti E, et al. A single cell resolution map of mouse haematopoietic stem and progenitor cell differentiation. Blood. 2016;128:20-32. 
47. Buenrostro JD, Corces MR, Lareau CA, Wu B, Schep AN, Aryee $\mathrm{MJ}$, et al. Integrated single-cell analysis maps the continuous regulatory landscape of human hematopoietic differentiation. Cell. 2018;173:1535-48.

48. Lai S, Huang W, Xu Y, Jiang M, Chen H, Cheng C, et al. Comparative transcriptomic analysis of hematopoietic system between human and mouse by Microwell-seq. Cell Discov. 2018;4:34.

49. Baron CS, Barve A, Muraro MJ, van der Linden R, Dharmadhikari G, Lyubimova A, et al. Cell type purification by single-cell transcriptome-trained sorting. Cell. 2019;179:527-42.

50. Xue Y, Liu D, Cui G, Ding Y, Ai D, Gao S, et al. A 3D atlas of hematopoietic stem and progenitor cell expansion by multidimensional RNA-Seq analysis. Cell Rep. 2019;27:1567-78.

51. Athanasiadis EI, Botthof JG, Andres H, Ferreira L, Lio P, Cvejic A. Single-cell RNA-sequencing uncovers transcriptional states and fate decisions in haematopoiesis. Nat Commun. 2017;8:2045.

52. Chen MJ, Yokomizo T, Zeigler BM, Dzierzak E, Speck NA. Runx1 is required for the endothelial to haematopoietic cell transition but not thereafter. Nature. 2009;457:887-91.

53. Zovein AC, Hofmann JJ, Lynch M, French WJ, Turlo KA, Yang Y, et al. Fate tracing reveals the endothelial origin of hematopoietic stem cells. Cell Stem Cell. 2008;3:625-36.

54. Kartalaei PS, Yamada-Inagawa T, Vink CS, de Pater E, van der Linden R, Marks-Bluth J, et al. Whole-transcriptome analysis of endothelial to hematopoietic stem cell transition reveals a requirement for Gpr56 in HSC generation. J Exp Med. 2015;212:93-106.

55. Davidson AJ, Zon LI. The "definitive" (and 'primitive') guide to zebrafish hematopoiesis. Oncogene. 2004;23:7233-46.

56. Svoboda O, Stachura DL, Macho ova O, Pajer P, Brynda J, Zon LI, et al. Dissection of vertebrate hematopoiesis using zebrafish thrombopoietin. Blood. 2014;124:220-8.

57. Sun H, Lesche R, Li DM, Liliental J, Zhang H, Gao J, et al. PTEN modulates cell cycle progression and cell survival by regulating phosphatidylinositol 3,4,5,-trisphosphate and Akt/ protein kinase B signaling pathway. Proc Natl Acad Sci USA. 1999;96:6199-204.

58. Reya T, Morrison SJ, Clarke MF, Weissman IL. Stem cells, cancer, and cancer stem cells. Nature. 2001;414:105-11.

59. Signer RAJ, Magee JA, Salic A, Morrison SJ. Haematopoietic stem cells require a highly regulated protein synthesis rate. Nature. 2014;509:49-54.

60. Challen GA, Boles N, Lin KKY, Goodell MA. Mouse hematopoietic stem cell identification and analysis. Cytom Part A. 2009;75:14-24.

61. Lin HT, Otsu M, Nakauchi H. Stem cell therapy: an exercise in patience and prudence. Philos Trans R Soc B Biol Sci. 2013;368:4-6. 